\title{
Method of Harmonic Source Identification Based on Harmonic Apparent Power
}

\author{
Lin $\mathrm{XU}^{1}$, Rui Xing $\operatorname{LIN}^{1}$, Shuang Ting XU 2,* \\ ${ }^{1}$ State Grid Sichuan Electric Power Research Institute, Chengdu, Sichuan, 610072, China \\ ${ }^{2}$ College of Electrical Engineering and Information Technology, Sichuan University, Chengdu, Sichuan, 610065, China
}

\begin{abstract}
Based on the harmonic apparent power defined by the IEEE 1459-2010 power standard, the paper proposed a new method of identifying the main harmonic source at the point of common coupling (PCC). Using the reference impedance method, the utility and customer harmonic voltage and current contributions are calculated. And then combined with the IEEE 1459-2010 standard, defined the harmonic apparent power caused by the independent action of the equivalent harmonic source on the system side and the user side, by directly comparing the size of the two to determine the location of the main harmonic source. The method can consider the comprehensive influence of harmonic voltage and harmonic current, then identify the main harmonic source at PCC point. Finally, the rationality of the method is verified by the simulation of the IEEE 13 node system.
\end{abstract}

\section{Introduction}

With the rapid development of modern industry and the gradual maturation of power electronics technology, a large number of nonlinear loads are connected to the power grid, injecting massive harmonic and affecting the power quality.

Identification the harmonic source correctly is the basis of harmonic control, and it have been a lot of research. Traditional harmonic active power method in [1], it may have a large error in that the result is influenced by the phase difference of the harmonic source. The method that identified harmonic source by the size of Budeanu distortion power [2], which did not set the threshold to distinguish between the small harmonic source and the non-pure resistance linear load. Based on load parameter segmentation, identifying whether the load is harmonic source by the degree of non-linearity in [3]. However, it is assuming that linear load as RL model caused the linear capacitive load be considered as harmonic source. Different non-active power component comparison method in [4], it compared the size of the fundamental reactive power $\mathrm{Q}_{1}$, Fryze reactive power $\mathrm{Q}_{\mathrm{F}}$ and Sharon reactive power apparent $\mathrm{S}_{\mathrm{Q}}$ to determine the main harmonic source. Then the team did further improving in [5], and introduced the fictitious reactive power $Q_{x}$ instead of $\mathrm{S}_{\mathrm{Q}}$. However, the accuracy of this method is not high when the system nominal power is small and the value of the fictitious reactive power $\mathrm{Q}_{\mathrm{x}}$ is between $\mathrm{Q}_{1}$ and $\mathrm{N}$.

\section{Methodology for Harmonic Source Identification}

\subsection{The Power Definition}

Under steady conditions, non-sinusoidal voltage and current can be decomposed as follows:

$$
\begin{aligned}
& v=v_{1}+v_{h} \\
& i=i_{1}+i_{h}
\end{aligned}
$$

Where $\mathrm{v}_{1}$ and $i_{1}$ are fundamental components, $\mathrm{v}_{\mathrm{h}}$ and $i_{h}$ are harmonic components, with the fundamental voltage phase as a reference, then

$$
\begin{aligned}
& v_{1}=\sqrt{2} V_{1} \sin w t \\
& v_{h}=\sqrt{2} \sum_{h \neq 1} V_{h} \sin \left(h w t+\alpha_{h}\right) \\
& i_{1}=\sqrt{2} I_{1} \sin \left(w t-\theta_{1}\right) \\
& i_{h}=\sqrt{2} \sum_{h \neq 1} I_{h} \sin \left(h w t+\beta_{h}\right)
\end{aligned}
$$

Where $\mathrm{h}$ is the number of harmonic, $\mathrm{V}_{1}$ and $\mathrm{V}_{\mathrm{h}}$ are the RMS value of fundamental voltage and the $h^{\text {th }}$ harmonic voltage, $I_{1}$ and $I_{h}$ are the RMS value of fundamental current and the $\mathrm{h}^{\text {th }}$ harmonic current, $\alpha_{h}$ and $\beta_{h}$ are the phase of the $\mathrm{h}^{\text {th }}$ harmonic voltage and current.

Apparent power can be decomposed as follows:

$$
\begin{aligned}
S^{2} & =V^{2} I^{2}=\left(V_{1} I_{1}\right)^{2}+\left(\mathrm{V}_{1} \mathrm{I}_{H}\right)^{2}+\left(\mathrm{V}_{H} \mathrm{I}_{1}\right)^{2}+\left(\mathrm{V}_{H} \mathrm{I}_{H}\right)^{2} \\
& =S_{1}{ }^{2}+D_{I}{ }^{2}+D_{V}{ }^{2}+S_{H}{ }^{2}
\end{aligned}
$$

Where $\mathrm{D}_{\mathrm{I}}, \mathrm{D}_{\mathrm{V}}, \mathrm{S}_{\mathrm{H}}$ are current distortion power, voltage distortion power and harmonic distortion power, respectively. This decomposition method was proposed by Professor Emanuel of Worcester Polytechnic Institute in [6], and adopted by the IEEE 1459-2010 
standard [7].

Combined with the definition of THD, the relationship between harmonic apparent power $\mathrm{S}_{\mathrm{H}}$ and THD is can be shown as:

$$
\begin{aligned}
S_{H} & =V_{H} I_{H}=V_{1} I_{1} \frac{V_{H} I_{H}}{V_{1} I_{1}} \\
& =S_{1} \mathrm{THD}_{\mathrm{V}} \mathrm{THD}_{\mathrm{I}}
\end{aligned}
$$

Where $T H D_{V}$ and $\mathrm{THD}_{\mathrm{I}}$ are the rate of voltage and current distortion, respectively. From the equation (5), harmonic apparent power $\mathrm{S}_{\mathrm{H}}$, as a part of apparent power, only contains the interaction of the nonfundamental voltage and current, therefore $S_{H}$ is not affected by fundamental components, which is the same with the harmonic distortion power. In addition, the harmonic apparent power is the result of each order of harmonic voltage and current. If the power component is taken as the key index for identifying the main harmonic source, the effects of each harmonic voltage and current can be comprehensively considered.

\subsection{The Reference Impedance}

According to reference impedance method proposed in [8], the harmonic contribution of utility and customer at PCC point can be obtained. The method can take into account the impact of impedance variation on the decomposition of contribution. The principle can be described as, setting a utility reference impedance and customer reference impedance, making the difference between the true impedance and the reference impedance equivalent as an additional harmonic source, and the original harmonic source plus additional harmonic source, is the new equivalent harmonic source. The specific process shown in Figure 1. Where $Z_{\mathrm{u}}$ and $Z_{\mathrm{c}}$ are harmonic impedance of utility and customer, $Z_{\mathrm{ur}}$ and $Z_{\mathrm{cr}}$ are the reference impedance of utility and customer, $V_{p c c}$ and $I_{p c c}$ are the voltage and current of PCC point.

According to figure 1, the new equivalent harmonic source of utility and customer after the conversion can be expressed as:

$$
\begin{gathered}
V_{u-\text { new }}=V_{p c c}-Z_{u r} I_{p c c} \\
I_{c-\text { new }}=V_{p c c} / Z_{c r}+I_{p c c}
\end{gathered}
$$

The harmonic reference impedance of utility $Z_{\mathrm{ur}}$ can be equivalent with the system short-circuit impedance, and the harmonic reference impedance of customer $Z_{\mathrm{cr}}$ can be equivalent with load resistance.
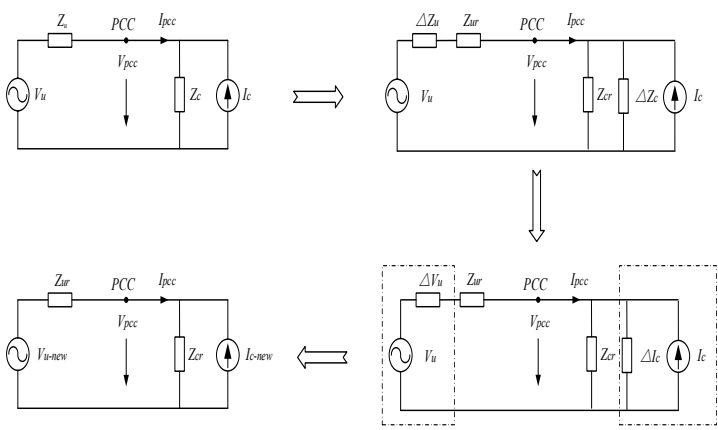

Fig. 1. The Diagram of Reference Impedance Method.

By using the superposition theorem, the harmonic voltage at PCC point caused by the independent action of the equivalent harmonic source on utility and customer is calculated with (7)

$$
\begin{aligned}
V_{p c c-u} & =\frac{Z_{c r}}{Z_{u r}+Z_{c r}} V_{u-n e w} \\
V_{p c c-c} & =\frac{Z_{u r} Z_{c r}}{Z_{u r}+Z_{c r}} I_{c-n e w}
\end{aligned}
$$

Similarly, the harmonic current at PCC point caused by the independent action of the equivalent harmonic source on utility and customer is calculated with (8)

$$
\begin{aligned}
I_{p c c-\mathrm{u}} & =\frac{1}{Z_{u r}+Z_{c r}} V_{u-\text { new }} \\
I_{p c c-c} & =-\frac{Z_{c r}}{Z_{u r}+Z_{c r}} I_{c-\text { new }}
\end{aligned}
$$

\subsection{Harmonic Source Identification}

The harmonic voltage and current at PCC point caused by the independent action of the equivalent harmonic source on utility are defined as $\mathrm{THD}_{\mathrm{v} u}$ and $\mathrm{THD}_{\mathrm{I} u}$, respectively.

$$
\mathrm{THD}_{\mathrm{V} u}=\frac{\sqrt{\sum_{h=2} V_{p c c h-u}^{2}}}{V_{p c c 1}} ; \operatorname{THD}_{\mathrm{I} u}=\frac{\sqrt{\sum_{h=2} I_{p c c h-u}^{2}}}{I_{p c c 1}}
$$

Where $V_{p c c 1}$ and $I_{p c c 1}$ are the RMS value of the fundamental voltage and current, respectively. $V_{\text {pcch-s }}$ and $\mathrm{I}_{\text {pcch-s }}$ are the RMS value of the $\mathrm{h}^{\text {th }}$ harmonic voltage and current at PCC point caused by the independent action of the utility harmonic source.

Similarly, the harmonic voltage and current at PCC point caused by the independent action of the customer are defined as $\mathrm{THD}_{\mathrm{V}_{c}}$ and $\mathrm{THD}_{\mathrm{Ic}}$, respectively.

$$
\begin{gathered}
T H D_{V c}=\frac{\sqrt{\sum_{h=2} V_{p c c h-c}^{2}}}{V_{p c c 1}} \\
T H D_{I c}=\frac{\sqrt{\sum_{h=2} I_{p c c h-c}^{2}}}{I_{p c c 1}}
\end{gathered}
$$

Where $V_{\text {pcc-c }}$ and $I_{\text {pcc-c }}$ are the RMS value of the $h^{\text {th }}$ harmonic voltage and current at PCC caused by the independent action of customer. Harmonic apparent 
powers $\mathrm{S}_{\mathrm{Hu}}$ and $\mathrm{S}_{\mathrm{Hc}}$ at $\mathrm{PCC}$ point caused by the the independent action of the equivalent harmonic source on the system side and the user side can be calculated with equation (12) and (13):

$$
\begin{aligned}
& S_{\mathrm{H} u}=S_{p c c 1} \operatorname{THD}_{\mathrm{V} u} \operatorname{THD}_{\mathrm{I} u} \\
& S_{\mathrm{H} c}=S_{p c c 1} \operatorname{THD}_{\mathrm{V} c} \operatorname{THD}_{\mathrm{I} c}
\end{aligned}
$$

Where $S_{\text {pccl }}$ is the fundamental apparent powers at PCC point.

In summary, the main harmonic source at $\mathrm{PCC}$ point can be identified by comparing the size of $\mathrm{S}_{\mathrm{Hs}}$ and $\mathrm{S}_{\mathrm{Hc}}$, the concrete process of the method are as follows:

(1) Measure voltage and current at PCC point, and analyze harmonic component

(2) Based on the reference impedance method, the reference impedance of utility and customer are equivalent to the system short circuit impedance and the load resistance, respectively. Then estimate the harmonic contribution of utility and customer that $\mathrm{V}_{\text {pcc- }}$ $\mathrm{u}, \mathrm{V}_{\mathrm{pcc}-\mathrm{c}}, \mathrm{I}_{\mathrm{pcc}-\mathrm{u}}, \mathrm{I}_{\mathrm{pcc}-\mathrm{c}}$, according to (6)-(8)

(3) According to (9)-(10), calculate the harmonic voltage and current at PCC point caused by the independent action of the utility and customer that $\mathrm{THD}_{\mathrm{V} u}, \mathrm{THD}_{\mathrm{I} u}, \mathrm{THD}_{\mathrm{V} c}, \mathrm{THD}_{\mathrm{Ic}}$.

(4) Calculate the harmonic apparent powers caused by the utility and customer harmonic source that $S_{\mathrm{H} u}$, $S_{\mathrm{Hc}}$, according to (11)-(12).

(5) Compare the size of $S_{\mathrm{H} u}$ and $S_{\mathrm{Hc}}$, if the former is larger, the main harmonic source is the upstream user side at the PCC that the utility. If the latter is larger, the main harmonic source is considered as the downstream user side at the PCC that the customer. If $S_{\mathrm{H} u}=S_{\mathrm{H} c}$, the utility and the customer contributions on the overall harmonic pollution at the measurement point are comparable.

\section{Case Studies}

In order to validate the performance of the proposed method, simulation tests are carried on the 13-bus IEEE test system [9] through PSCAD/EMTDC, as shown in figure 2, and simulation data are analyzed through MATLAB. In this test system, five measurement points are selected as the PCC of interest: 32, 33, 45, 92 and 84.

The test system is powered by the node 50 , and it includes voltage regulators, single-phase and three-phase loads. The harmonic source loads include the fluorescent lamp group, the frequency converter (ASD) and other household loads. The system reference impedance can be determined by the system short-circuit impedance.

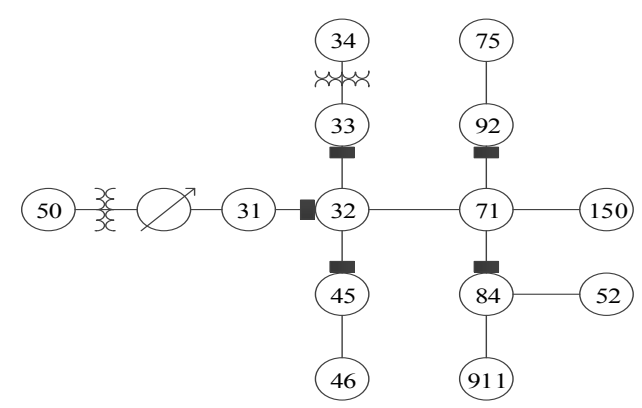

Fig. 2. The 13-bus IEEE test system.

\section{A. Case 1}

The harmonic source is connected to node 75 and node 92 , the proposed method and the non-active power comparison method [8] are applied at each measurement point, and the results are shown in Table 1 and Table 2. At this case, the actual main harmonic source of node 32 and node 92 is downstream of the measurement point (the customer side), and the rest of the measurement points are the upstream of the measurement point (the utility side).

Tab. 1. The Results of Case 1

(Non-active Power Comparison Method)

\begin{tabular}{ccccc}
\hline & $Q_{1} /$ kvar & $Q_{x} /$ kvar & $N /$ kvar & Result \\
\hline 32 & 1027.45 & 1034.66 & 1040.75 & Customer \\
33 & 178.43 & 178.46 & 178.69 & Utility \\
45 & 229.38 & 229.41 & 229.43 & Utility \\
84 & 10.47 & 11.99 & 18.46 & Utility \\
92 & 381.6 & 405.83 & 426.7 & Customer \\
\hline
\end{tabular}

Tab. 2. The Results of Case 1 (Proposed Method)

\begin{tabular}{cccc}
\hline & $S_{\mathrm{Hs}} / \mathrm{kVA}$ & $S_{\mathrm{Hc}} / \mathrm{kVA}$ & Result \\
\hline 32 & 0 & 2.88 & Customer \\
33 & 0.14 & 0 & Utility \\
45 & 0.05 & 0 & Utility \\
84 & 0.26 & 0 & Utility \\
92 & 0 & 5.33 & Customer \\
\hline
\end{tabular}

According to Table 1 and Table 2, it shows that in the proposed method, the value of $\mathrm{S}_{\mathrm{Hc}}$ in node 33, 45 and 84 are approximate to 0 , far less than the value of $\mathrm{SHu}$, so identified the upstream of measurement point (the utility side) as the main harmonic source identification for measurement points (the system side), and the utility side should responsible for harmonic pollution of the three measuring points. The value of $\mathrm{S}_{\mathrm{Hc}}$ in node 32 and 92 are greater than the value of $\mathrm{SHu}$, and $\mathrm{SHu}$ value is approximately equal to 0 , therefore identified downstream of the measuring point (the customer side) is the main harmonic source, and the customer side should take full responsibility for the harmonic pollution of the two measurement points. The proposed method in all measurement points are given the correct result.

In the non-active power comparison method, the fictitious reactive power Qx in node 32 and node 92 are closer to non-active power $\mathrm{N}$, therefore can identified downstream of the measuring point (the customer side) as the main harmonic source. For the remaining measurement points, since the fictitious reactive power Qx 
is closer to the fundamental reactive power $\mathrm{Q}_{1}$, the upstream of the measuring point (the utility side) is identified as the main harmonic source. This method also gives correct results at all measurement points.

\section{B. Case 2}

The harmonic source is connected to node $75,92,45,46$, and the simulation results are listed in Table 3 and Table 4. At this case, the actual main harmonic source of node 32,45 and 92 is downstream of the measurement point (the customer side), and the rest of the measurement points are the upstream of the measurement point (the utility side).

Tab. 3. The Results of Case 2

(Non-active Power Comparison Method)

\begin{tabular}{ccccc}
\hline & $Q_{1} /$ kvar & $Q_{x} /$ kvar & $N /$ kvar & Result \\
\hline 32 & 1072.66 & 1080.86 & 1086.01 & Customer \\
33 & 178.30 & 178.34 & 178.57 & Utility \\
45 & 266.07 & 226.09 & 278.16 & Utility \\
84 & 12.63 & 14.80 & 17.69 & Utility \\
92 & 376.98 & 401.20 & 422.24 & Customer \\
\hline
\end{tabular}

Tab. 4. The Results of Case 2

\begin{tabular}{cccc}
\multicolumn{4}{c}{ (Proposed Method) } \\
\hline & $S_{\mathrm{Hs}} / \mathrm{kVA}$ & $S_{\mathrm{Hc}} / \mathrm{kVA}$ & Result \\
\hline 32 & 0 & 2.74 & Customer \\
33 & 0.14 & 0 & Utility \\
45 & 0.25 & 0.59 & Customer \\
84 & 0.16 & 0 & Utility \\
92 & 2.08 & 3.13 & Customer \\
\hline
\end{tabular}

In the non-active power comparison method, since the values of the three powers are very close, it can be considered that there is no non-linear load at the downstream of the measurement point 33, which is consistent with the fact. The fictitious reactive power Qx is closer to the fundamental reactive power Q1 than to the non-active power $\mathrm{N}$ in node 45 , therefore given a false result that does not conform to the facts.

For the proposed method, the value of $\mathrm{S}_{\mathrm{Hs}}$ at measurement point 32 is about equal zero, so the downstream of the measurement point (the customer side) is fully responsible for harmonic pollution. Similarly, at measurement points 33 and 84, the value of $\mathrm{SHu}$ is approximate to zero, so the upstream of the measurement point are main harmonic source. For the measurement points 45 and 92, since the value of $\mathrm{S}_{\mathrm{Hc}}$ is larger than $\mathrm{SHu}$, the main harmonic source is the downstream of this measurement point (the customer side), which is consistent with the fact that actual harmonic sources are injected into the system.

\section{Conclusion}

In this paper, a method of main harmonic source identification is proposed based on harmonic apparent power. According to the reference impedance method, the harmonic voltage and current at the PCC point when the utility and the customer function separately are calculated, and it can avoid the influence of the impedance variation on the contribution decomposition. Based the harmonic apparent power component in IEEE 1459-2010, a new definition of harmonic apparent power $\mathrm{S}_{\mathrm{Hu}}$ and $\mathrm{S}_{\mathrm{Hc}}$ which generated when the utility and customer individual action is proposed, and identified the main harmonic source by comparing the size of the two. Simulation analysis verifies the effectiveness of the proposed method.

The proposed method starts from the view of the apparent power decomposition, and consider not only the harmonic voltage or current of a concern order, but also the comprehensive influence of various harmonic order voltage and current, which provides a new way to distinguish the comprehensive harmonic pollution responsibility between the the utility side and the customer side. The method proposed in this paper is simple in operation and can be easily applied to the metering device, which is of engineering significance.

\section{Acknowledgement}

The work was supported by project: Research on Harmonic Quantization Method for Nonlinear Loads(NO: 52199716001P), State Grid Sichuan Electric Power Research Institute.

\section{References}

1. Yang H, Porotte P, Robert A. Proceedings of the 3rd International Conference on Power Quality. 2, 160 (1994)

2. D Stevanović, P Petković. Electrical Engineering. 97, $165(2015)$

3. Antonio Dell'Aquila, Maria Marinelli. IEEE Transactions on Power Delivery. 19, 1284 (2004)

4. Cataliotti A, Cosentino V, Nuccio S. IEEE Transactions on Instrumentation and Measurement. 57, 1554 (2008)

5. Cataliotti A, Cosentino V, Nuccio S. IEEE Transactions on Instrumentation and Measurement. 57, 85 (2008)

6. Emanuel A E. IEEE Transactions on Instrumentation and Measurement. 3, 1457 (2005)

7. IEEE Standard definitions for the measurement of electric power quantities under sinusoidal , nonsinusoidal , balanced , or unbalanced conditions. IEEE Std 1459, 2010.

8. Tomaž Pfajfar, Boštjan Blažip, Igor Papip. 9th International Conference on Electrical Power Quality and Utilization(2007).

9. IEEE Working Group on Nonsinusoidal Situations. IEEE Transactions on Power Delivery. 11, 79 (1996) 